Ophthalmologe 2015 · 112:319-324

DOI 10.1007/s00347-014-3163-4

Online publiziert: 3. April 2015

(c) Springer-Verlag Berlin Heidelberg 2015

M.C. Grieshaber

Universitäts-Augenklinik Basel

\title{
Kanalographie und Wirkmechanismus der Kanaloplastik
}

natürlichen KWAS wieder ins Zentrum, weil ein intaktes oder wiederhergestelltes KWAS distal des Trabekelmaschenwerkes (TM) eine wichtige Voraussetzung ist, damit die Kanaloplastik funktioniert [11, 12]. Neue bildgebende Verfahren wie die optische Kohärenztomographie oder die hochauflösende Ultraschallbiomikroskopie mögen zwar gute Aufnahmen des vorderen Augenabschnittes geben, sind aber nicht in der Lage, Informationen über den Funktionszustand des KWAS zu liefern. Deshalb haben wir 2 klinische Tests, die provokative Gonioskopie und die Kanalographie, beschrieben, die dem Chirurgen helfen sollen, den Zustand des KWAS vor und während der Operation besser zu beurteilen und den Erfolg einer Kanaloplastik abzuschätzen [7, 8]. Wir haben hierzu das KWAS in das proximale (TM) und distale (Schlemm-Kanal und Sammelkanäle) Abflusssystem unterteilt.

\section{Provokative Gonioskopie}

Bei der provokativen Gonioskopie wird der Augendruck unter den episkleralen Venendruck nach Parazentese und Aspiration des Kammerwassers aus der Vorderkammer gesenkt. Durch die okuläre Hypotonie wird eine Druckumkehr mit natürlichem Blutreflux aus den vorderen Ziliarvenen über die Sammelkanäle in den Schlemm-Kanal erzeugt. Nach 1 min Wartezeit wird ein Kontaktglas (CGA 1, Haag Streit, Bern, Schweiz) auf das Auge platziert und das Muster des Blutrefluxes im Schlemm-Kanal beobachtet und klassifiziert. Im Rahmen einer Studie haben wir 3 Füllungsmuster festgestellt:
- kein Blutreflux,

- partielle respektive unvollständige und

- vollständige Blutfüllung des Schlemm-Kanals [8].

Eine fehlende Füllung deutet auf einen vollständig kollabierten Schlemm-Kanal und atrophierte oder zumindest verschlossene Ostien der Sammelkanäle hin. Eine segmentale Blutfüllung entspricht einem partiellen Kollaps des Kanallumens mit noch durchgängigen Sammelkanälen, und eine vollständige Füllung bei intaktem, offenem Kanalsystem ist als homogenes kontinuierliches Band sichtbar (• Abb. 1, 2).

》) Die Qualität des Blutrefluxes korreliert umgekehrt mit der Höhe des Augeninnendrucks

Kürzlich konnten wir bei Patienten mit einem primären Offenwinkelglaukom unterschiedliche Muster von Blutreflux feststellen, die für den Operationserfolg einer Kanaloplastik prognostisch waren. Die Qualität des Blutrefluxes korrelierte umgekehrt mit der Höhe des Augeninnendrucks vor der Operation - unabhängig vom Alter der Patienten: Je höher der Augeninnendruck war, desto geringer der Blutreflux. So war der Blutreflux homogen und vollständig bei Augen mit einem Augeninnendruck von etwa $30 \mathrm{mmHg}$, partiell bei Augen mit einem Augeninnendruck von $40 \mathrm{mmHg}$ und fehlend bei Augen mit einem Augeninnendruck über $50 \mathrm{mmHg}$. Diejenigen Augen mit dem 


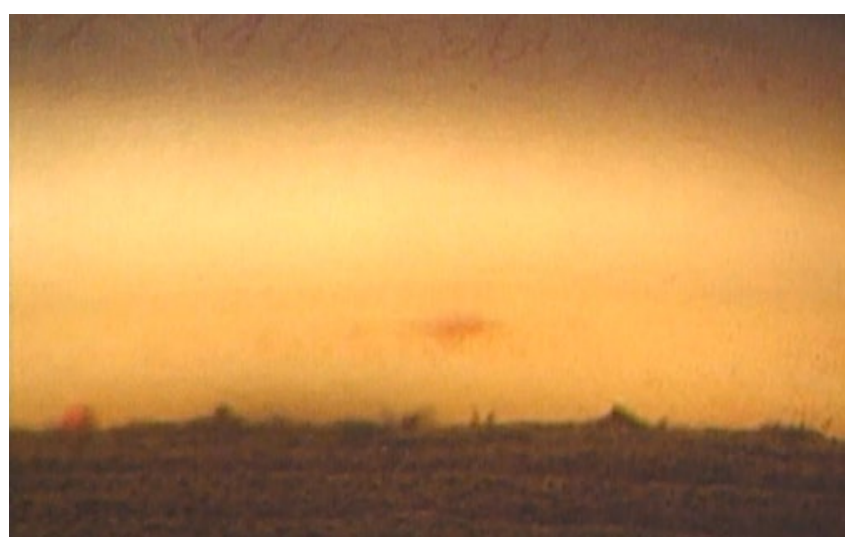

Abb. $1 \Delta$ Fehlender Blutreflux in den Schlemm-Kanal. Es ist nur wenig Blut am Ostium des Sammelkanals sichtbar. Dies deutet auf einen kollabierten Kanal hin

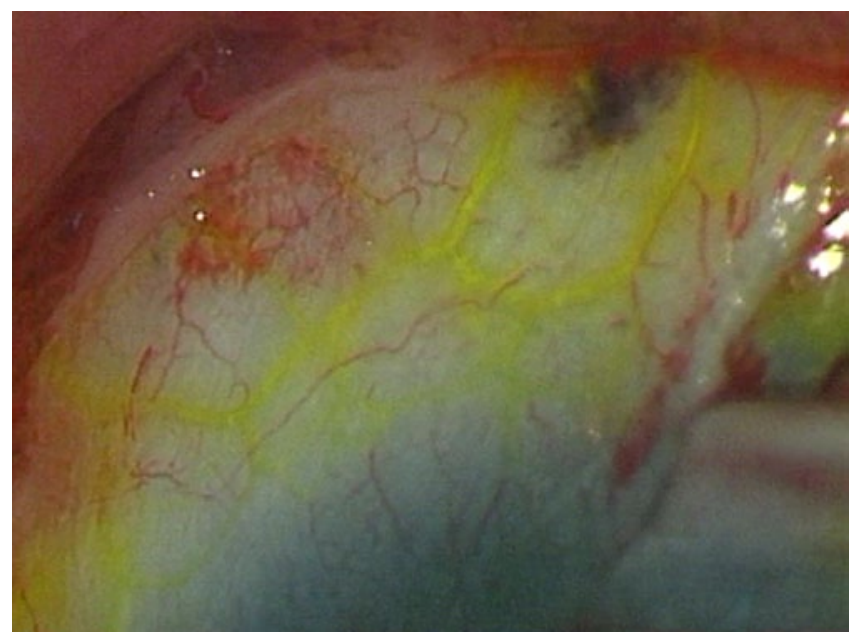

Abb. $3 \Delta$ Die Füllung der episkleralen Venen ist bei diesem Auge in der Kanalographie gut sichtbar, die Diffusion in die Vorderkammer jedoch schlecht. In diesem Fall muss von einer proximalen Abflussstörung ausgegangen werden

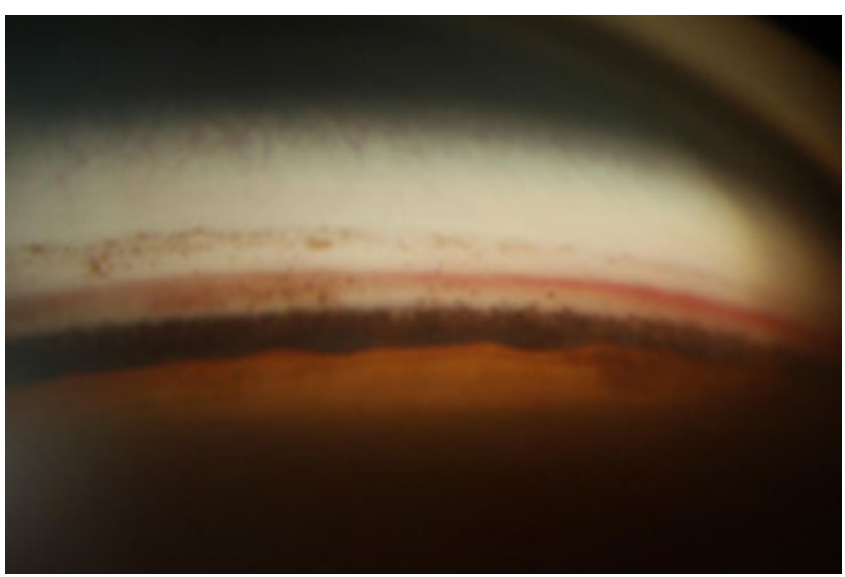

Abb. $2 \Delta$ Homogene und vollständige Füllung des Schlemm-Kanals mit Blut als Zeichen eines offenen Kanals

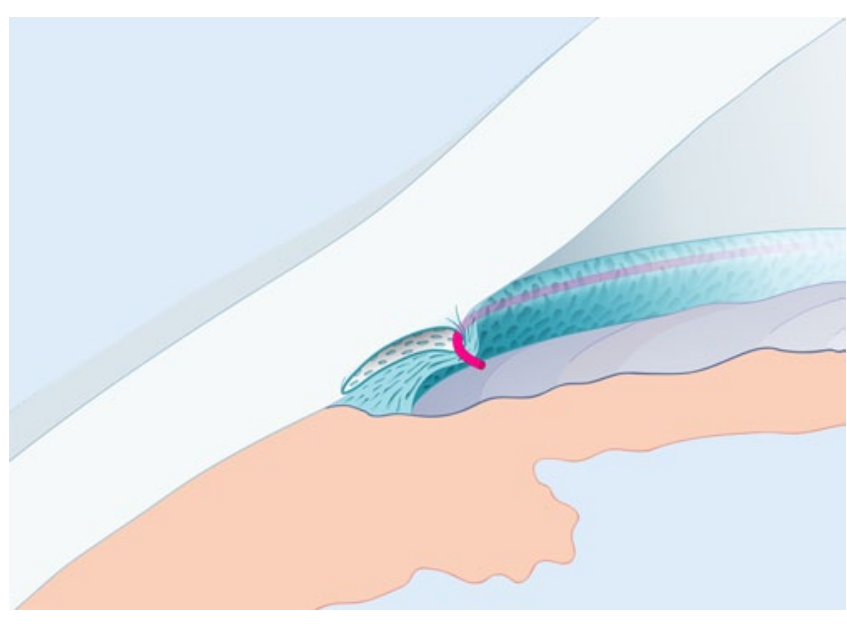

Abb. 4 A Schematische Darstellung: Der Spannungsfaden dehnt die innere Wand des Schlemm-Kanals und erhöht so die Durchlässigkeit des Trabekelmaschenwerks und damit den Kammerwasserabfluss geringsten Blutreflux hatten die geringste Drucksenkung nach der Kanaloplastik [8]. Anzufügen ist hier, dass diese Patienten keine Langzeittherapie mit drucksenkenden Augentropfen vor der Operation hatten, welche die Morphologie des KWAS beeinflussen könnten.

Die provokative Gonioskopie hat gegenüber früheren Methoden den Vorteil [15, $16,17,18,22]$, dass der Blutreflux nicht durch Kompression der episkleralen Venen durch das Gonioskop erzeugt wird, welche fälschlicherweise das Lumen der Sammelkanäle und des Schlemm-Kanals verändert und somit den ursprünglichen Zustand dieser falsch wiedergibt, sondern durch eine okuläre Hypotonie als Folge einer Umkehr des Druckgradienten zwischen dem episkleralen Venen- druck und dem Augendruck nach Parazentese.

\section{Kanalographie}

Unter der Kanalographie versteht man die Darstellung des Schlemm-Kanals, der episkleralen Venen sowie die transtrabekuläre Diffusion von verdünntem Fluoreszeinfarbstoff in die Vorderkammer $[7,8]$. Nach Eröffnung des Schlemm-Kanals wird ein Mikrokatheter (iTrack 250, iScience Interventional, Menlo Park, USA) schrittweise in den Kanal eingeführt und verdünntes Fluoreszeinsodium an verschiedenen Orten injiziert. Wie in einer früheren Studie gezeigt, gilt die Diffusion mit Fluoreszein als Indikator für die Permeabilität des TM einerseits und die Füllung als Durchgängigkeit der episkleralen Venen andererseits. Ein Großteil der Patienten hatte keine oder eine verzögerte Diffusion, dies als Zeichen einer verminderten Permeabilität des erkrankten TM. Jedoch war die Höhe des Augeninnendrucks bezüglich Füllung der episkleralen Gefäße nicht aufschlussreich. Dabei ist zu berücksichtigen, dass ein erhöhter Augeninnendruck nur ein Surrogat für einen erhöhten Widerstand beim Offenwinkelglaukom ist - ohne Hinweis für die Lokalisation im KWAS. Klinisch und im Einzelfall deuten eine schlechte Diffusion in die Vorderkammer und eine gute Venenfüllung primär auf eine Obstruktion im proximalen Abflusssystem (TM), während eine gute Diffusion bei schlechter Venenfüllung Hinweise für eine Ein- 


\section{Hier steht eine Anzeige.}

算 Springer 
schränkung im distalen Abflusssystem (Schlemm-Kanal und Sammelkanäle) liefert (• Abb. 3).

Die Darstellung des Abflusssystems mit Fluoreszein ist nicht neu und wurde schon von Wessely [25] 1922 erstmals beschrieben. Seither haben viele Forscher das KWAS mittels Fluoreszeininjektion in die Vorderkammer direkt untersucht [2, 5, 19, 24]. Der Vorteil jener Methode ist, dass das Fluoreszein entlang der natürlichen Flussrichtung von der Vorderkammer durch das TM in den Schlemm-Kanal gelangt. Der Nachteil ist aber die relativ hohe Konzentration von Fluoreszein, weil der Farbstoff unverzüglich mit Kammerwasser vermischt und verdünnt wird. Im Weiteren verzögert die langsame Diffusion durch das TM die Füllung der episkleralen Venen, welche die Beurteilung des distalen KWAS einschränkt. Deshalb erkannte Grote [9] schon früh den Vorteil der Injektion von Fluoreszein direkt in den Schlemm-Kanal. Während der Trabekulotomie injizierte er Fluoreszein am Operationszugang und versuchte den ganzen Kanal zirkulär zu füllen. Dazu benötigte er große Volumina und hohe Konzentrationen an Fluoreszein. Unsere Methode hat den großen Vorteil, dass mithilfe des Mikrokatheters und der eingebauten Lichtsonde verschiedene Orte des KWAS mit geringen Fluoreszeinmengen gezielt, direkt und unabhängig voneinander untersucht werden können.

\section{》) Ein intaktes peripheres}

\section{Kammerwasserabfluss- \\ system ist Voraussetzung \\ für die Kanaloplastik}

Aus den beschriebenen Testverfahren lässt sich ableiten, dass ein intaktes peripheres KWAS eine essenzielle Voraussetzung dafür ist, dass mit der Kanaloplastik eine wirksame Drucksenkung erzielt werden kann. Erweist sich der Abfluss aus diesen Tests als eingeschränkt und ungenügend, sollte der Chirurg als praktische Konsequenz ein filtrierendes (penetrierendes oder nicht penetrierendes) Verfahren mit Umgehung des KWAS wählen, wie z. B. eine tiefe Sklerektomie. Ebenso sehr ist ein Verfahren mit subkonjunktivalem Abfluss nach ungenügender

Ophthalmologe 2015 · 112:319-324 DOI 10.1007/s00347-014-3163-4

๑) Springer-Verlag Berlin Heidelberg 2015

\section{M.C. Grieshaber \\ Kanalographie und Wirkmechanismus der Kanaloplastik}

\section{Zusammenfassung}

Hintergrund. Die Kanaloplastik stellt das physiologische Kammerwasserabflusssystem im Auge chirurgisch wieder her. Der Operationserfolg hängt von dem Funktionszustand dieses Systems ab.

Ziel der Arbeit. Untersucht wurden der Stellenwert des Kammerwasserabflusssystems und der Wirkmechanismus der Kanaloplastik. Zwei klinische Tests, die provokative Gonioskopie und die Kanalographie, werden zur Beurteilung des Kammerwasserabflusssystems beschrieben.

Material und Methoden. Bei der provokativen Gonioskopie wird durch Senkung des Augendruckes mittels Kammerwasseraspiration eine Druckumkehr zwischen dem Augendruck und dem episkleralen Venendruck erzielt, und der Blutreflux wird klassifiziert. Die Kanalographie untersucht die Qualität der Fluoreszeindiffusion durch das Trabekelmaschenwerk und die Füllung der episkleralen Venen.

Ergebnisse. Der Blutreflux variiert bei Glaukomaugen und korreliert umgekehrt mit dem Augendruck vor der Operation. Je höher der Augendruck war, desto schlechter die Blutfüllung im Kanal. Die Füllung der episkleralen Venen und die Fluoreszeindiffusion in die Vorderkammer waren unterschiedlich. Eine schlechte Diffusion in die Vorderkammer und eine gute Venenfüllung weisen auf eine proximale Abflussstörung im Trabekelmaschenwerk, eine schlechte Diffusion und eine schlechte Venenfüllung auf eine proximale und distale Abflussstörung und eine gute Diffusion bei einer schlechten Venenfüllung auf eine distale Abflussstörung im Schlemm-Kanal und den Sammelkanälen.

Schlussfolgerung. Das Blutrefluxmuster, das Ausmaß der Füllung des episkleralen Venensystems und der transtrabekulären Diffusion geben klinische Hinweise auf den funktionellen Zustand des Kammerwasserabflusssystems und auf den Erfolg der Kanaloplastik. Der Wirkmechanismus der Kanaloplastik ist bis heute nicht ganz geklärt. Diskutiert werden die $360^{\circ}$-Viskodilatation, eine dauerhafte Dehnung der inneren Kanalwand durch den Spannungsfaden oder den Stegmann-Kanalexpander.

\section{Schlüisselwörter}

Gonioskopie · Augeninnendruck .

Kammerwasserabfluss · Sammelkanäle .

Blutreflux

\section{Channelography and mechanism of action in canaloplasty}

\section{Abstract}

Background. Canaloplasty lowers the intraocular pressure (IOP) by restoring the natural outflow system. The success of canaloplasty depends on the function of this system.

Objectives. To evaluate the natural outflow system regarding canaloplasty by two clinical tests, provocative gonioscopy and channelography and to describe the mechanism of action of canaloplasty.

Material and methods. Provocative gonioscopy evaluates the pattern of blood reflux which is induced by ocular hypotension as the result of a reversed pressure gradient between the episcleral venous pressure and IOP following paracentesis. In channelography the transtrabecular diffusion and the filling properties of the episcleral venous system are assessed by a microcatheter and a fluorescein tracer.

Results. Blood reflux varied greatly in glaucomatous eyes and showed an inverse correlation with the preoperative IOP. The higher the IOP, the poorer the blood reflux. The filling qualities of the episcleral venous system and diffusion through the trabecular mesh- work were different. Poor trabecular passage and good episcleral fluorescein outflow indicates patent distal outflow pathways, poor trabecular passage and poor episcleral fluorescein outflow indicates obstructed trabecular meshwork and closed collector channels and good trabecular passage together with poor episcleral fluorescein outflow suggests that the site of impairment is mainly in the distal outflow system.

Conclusions. The quality of blood reflux and the characteristics of the episcleral filling and the transtrabecular diffusion by fluorescein represent the clinical state of the outflow pathway and help in the prediction of the surgical outcome in canaloplasty. The mechanism for canaloplasty is not yet completely clarified; currently under discussion are circumferential viscodilation, permanent distension of the inner wall of Schlemm's canal using a suture and a Stegmann canal expander.

\section{Keywords}

Gonioscopy · Intraocular pressure · Outflow system · Collector channels - Blood reflux 
Drucksenkung am zweiten Auge (Partnerauge) zu wählen.

\section{Wirkmechanismus der Kanaloplastik}

Auch wenn die Kanaloplastik in verschiedenen Studien ihre Wirksamkeit bewiesen hat, ist relativ wenig über den Wirkmechanismus bekannt. Die Kanaloplastik besteht aus 2 chirurgischen Teilschritten, der $360^{\circ}$-Kanülierung mit Viskodilatation des Schlemm-Kanals und der Dehnung der inneren Kanalwand und des TM durch einen 10-0-Prolene-Faden oder einen Stent (Stegmann Canal Expander, Ophthalmos GmbH, Schaffhausen, Schweiz; $\bullet$ Abb.4). Wie aus experimentellen Studien an Primaten und menschlichen Autopsieaugen gezeigt, führt die Injektion von Viskoelastikum in den Schlemm-Kanal nicht nur zu einer Erweiterung des Kanallumens um etwa das 10-Fache von 25 auf $230 \mu \mathrm{m}$, sondern auch zu Mikrorissen der inneren und äußeren Kanalwand [20, 21, 23, 28]. Dies stellt eine direkte Verbindung zwischen der Vorderkammer und dem Lumen des Schlemm-Kanals sowie des choroidalen Gewebe dar, das wiederum den konventionellen und uveoskleralen Ausfluss erhöht. Da Viskoelastikum normalerweise nach $48-72 \mathrm{~h}$ aus dem Schlemm-Kanal ausgewaschen wird, ist die Erweiterung des Schlemm-Kanals bei der Viskokanalostomie nur von kurzer Dauer. Dennoch werden mit der Viskokanalostomie langfristig akzeptable Druckwerte im oberen Normbereich erzielt $[6,26,27]$.

Im Gegensatz zur Viskokanalostomie, die den Schlemm-Kanal nur für etwa ein Drittel in der Länge verändert (ca. $120^{\circ}$ ), geschieht dies mit der Kanaloplastik zirkulär $\left(360^{\circ}\right)$. Dabei werden die Diffusion durch das TM und der direkte Abfluss zu den Sammelkanälen fern der Eröffnung des Schlemm-Kanals (DescemetFenster) verbessert. Zu berücksichtigen ist auch die Tatsache, dass die meisten Sammelkanäle im unteren und nasalen Quadranten liegen $[1,13,21]$. In einer Pilotstudie an perfundierten Autopsieaugen zeigte die Erweiterung von $180^{\circ}$ mit dem Mikrokatheter einen verbesserten Abfluss und eine größere Augendrucksenkung, die in direktem Bezug zur Menge der Viskodi- latation stand [10]. Ebenso führte eine alleinige zirkuläre Viskodilatation zu einer Drucksenkung in den mittleren bis oberen Zehnerbereich [3]. Die permanente Fadenspannung, die einen zirkumferenten Kanalfluss ermöglicht und das Risiko des Kollabierens des Schlemm-Kanals vermindert, wird als weiterer Teil des Wirkmechanismus der Kanaloplastik angesehen. Zurzeit ist jedoch unklar, wie viel jeder der postulierten Faktoren zum verbesserten Kammerwasserabfluss und damit zur Augeninnendrucksenkung beiträgt: die Bildung des Sklerabettes und des Descemet-Fensters, die $360^{\circ}$-Dilatation und die Fadenspannung. Einige Studien haben die Fadenspannung als Dehnungswinkel der inneren Kanalwand mit der Ultraschallbiomikroskopie untersucht $[4,14]$. Es scheint, dass je größer die Fadenspannung ist, desto tiefer der Augendruck nach der Operation. Unsere eigenen Ergebnisse konnten einen direkten Zusammenhang zwischen der Fadenspannung und dem postoperativen Augendruck jedoch nicht bestätigen. Zu berücksichtigen ist, dass der Dehnungswinkel der inneren Kanalwand nur indirekt die Fadenspannung wiedergibt und strukturelle Veränderungen nicht mit funktionellen korrelieren müssen.

\section{Fazit für die Praxis}

- Die Kanaloplastik ist eine wirksame Glaukomoperation, die filterkissenunabhängig den Augeninnendruck senkt, indem sie versucht das physiologische KWAS wiederherzustellen.

- Der Operationserfolg hängt von der Durchgängigkeit des proximalen (TM) und des distalen (Schlemm-Kanal und Sammelkanäle) Systems ab.

- Die Intaktheit des KWAS kann mithilfe der provokativen Gonioskopie und der Kanalographie klinisch beurteilt werden.

- Bei eingeschränkter Funktion des KWAS ist ein filtrierendes Verfahren mit Ausbildung eines Filterkissens, wie z. B. die tiefe Sklerektomie, zu wählen.

- Zurzeit wird ein 3-facher Wirkmechanismus postuliert: $360^{\circ}$-Viskodilatation mit Mikrorupturen der inneren und äußeren Kanalwand, dauerhaf- te Dehnung der inneren Kanalwand durch den Spannungsfaden oder den Stegmann-Kanalexpander sowie Bildung des Sklerabettes und Descemet-Fensters. Wie viel jeder einzelne Schritt dazu beiträgt, muss noch in zukünftigen Studien untersucht werden.

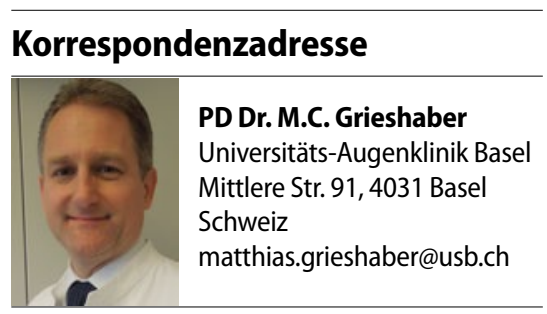

\section{Einhaltung ethischer Richtlinien}

Interessenkonflikt. M.C. Grieshaber hat ein gewerbliches Eigentumsrecht am Stegmann Canal Expander.

Dieser Beitrag beinhaltet keine Studien an Menschen oder Tieren.

\section{Literatur}

1. Ashton N (1951) Anatomical study of Schlemm's canal and aqueous veins by means of neoprene casts. Part I. Aqueous veins. Br J Ophthalmol 35:291-303

2. Benedikt O (1976) Demonstration of aqueous outflow patterns of normal and glaucomatous human eyes through the injection of fluorescein solution in the anterior chamber (author's transl). Albrecht Von Graefes Arch Klin Exp Ophthalmol 199:45-67

3. Cameron B, Field M, Ball S, Kearney J (2006) Circumferential viscodilation of Schlemm's canal with a flexile microcannula during non-penetrating glaucoma surgery. Digit J Ophthalmol 12(1). Zugegriffen: 15. März 2006

4. Doro D, Koerber N, Paolucci P et al (2010) Ultrasound biomicroscopy after canaloplasty: clinical study with two different units. Acta Clin Croat 51(Suppl 1):113-118

5. Goldmann H (1949) Die Kammerwasservenen und das Poisseuille'sche Gesetz. Ophthalmologica 118:496-519

6. Grieshaber MC, Peckar C, Pienaar A et al (2014) Long-term results of up to 12 years of over $700 \mathrm{ca}$ ses of viscocanalostomy for open-angle glaucoma. Acta Ophthalmol. doi: 10.1111/aos.12513 [Epub ahead of print

7. Grieshaber MC, Pienaar A, Olivier J et al (2009) Channelography: imaging of the aqueous outflow pathway with flexible microcatheter and fluorescein in canaloplasty. Klin Monatsbl Augenheilkd 226:245-248

8. Grieshaber MC, Pienaar A, Olivier J et al (2010) Clinical evaluation of the aqueous outflow system in primary open-angle glaucoma for canaloplasty. Invest Ophthalmol Vis Sci 51:1498-1504 
9. Grote $P(1978)$ Indications for the location of trabeculotomy in simple glaucoma. II. The demonstration of aqueous outflow with fluorescein. Doc Ophthalmol 46:171-183

10. Hee M, Conston S, Yamamoto R (2004) Outflow facility after 180 degree catheterization and distal expansion of Schlemm's canal perfused cadaver eyes [abstract]. ARVO Poster \# 984

11. Johnson DH, Johnson M (2002) Glaucoma surgery and aqueous outflow: how does nonpenetrating glaucoma surgery work? Arch Ophthalmol 120:67-70

12. Johnson DH, Johnson M (2001) How does nonpenetrating glaucoma surgery work? Aqueous outflow resistance and glaucoma surgery. J Glaucoma 10:55-67

13. Kleinert $H$ (1953) Vital staining of aqueous humor and its epibulbar outflow pathways following fluorescein injection into anterior chamber. Klin Monatsbl Augenheilkd Augenarztl Fortbild 122:665682

14. Lewis RA, Von Wolff K, Tetz M et al (2011) Canaloplasty: three-year results of circumferential viscodilation and tensioning of Schlemm canal using a microcatheter to treat open-angle glaucoma. J Cataract Refract Surg 37:682-690

15. Moses RA (1979) Circumferential flow in Schlemm's canal. Am J Ophthalmol 88:585-591

16. Phelps CD, Asseff CF, Weisman RL et al (1972) Blood reflux into Schlemm's canal. Arch Ophthalmol 88:625-631

17. Schirmer KE (1971) Gonioscopic assessment of blood in Schlemm's canal. Correlation with glaucoma tests. Arch Ophthalmol 85:263-267

18. Schirmer KE (1969) Reflux of blood in the canal of Schlemm, quantitated. Can J Ophthalmol 4:40-44

19. Schulte D (1948) Erfahrungen bei der Beobachtung von Wasservenen. Verh Dtsch Ophthalmol Ges 54:242-344

20. Smit BA, Johnstone M (2000) Effects of viscocanalostomy on the histology of Schlemm's canal in primate eyes. Invest Ophthalmol Vis Sci 41(4):S578

21. Smit BA, Johnstone MA (2002) Effects of viscoelastic injection into Schlemm's canal in primate and human eyes: potential relevance to viscocanalostomy. Ophthalmology 109:786-792

22. Suson EB, Schultz RO (1969) Blood in Schlemm's canal in glaucoma suspects. A study of the relationship between blood-filling pattern and outflow facility in ocular hypertension. Arch Ophthalmol 81:808-812

23. Tamm ER, Carassa RG, Albert DM et al (2004) Viscocanalostomy in rhesus monkeys. Arch Ophthalmol 122:1826-1838

24. Thomassen TL, Perkins ES, Dobree JH (1950) Aqueous veins in glaucomatous eyes. $\mathrm{Br} \mathrm{J}$ Ophthalmol 34:221-227

25. Wessely K (Hrsg) (1922) Die physiologischen und anatomischen Grundlagen der neueren Glaukomoperationen. Karger, Berlin

26. Wishart PK, Wishart MS, Choudhary A et al (2008) Long-term results of viscocanalostomy in pseudoexfoliative and primary open angle glaucoma. Clin Exp Ophthalmol 36:148-155

27. Wishart PK, Wishart MS, Porooshani H (2003) Viscocanalostomy and deep sclerectomy for the surgical treatment of glaucoma: a longterm followup. Acta Ophthalmol Scand 81:343-348

28. Grieshaber MC, Stegmann R, Grieshaber HR, Meyer P (2015) Novel device for expanding Schlemm's canal: a morphological study. Br J Ophthalmol. doi: 10.1136/bjophthalmol-2014-30554 [Epub ahead of print]

\section{Fachnachrichten}

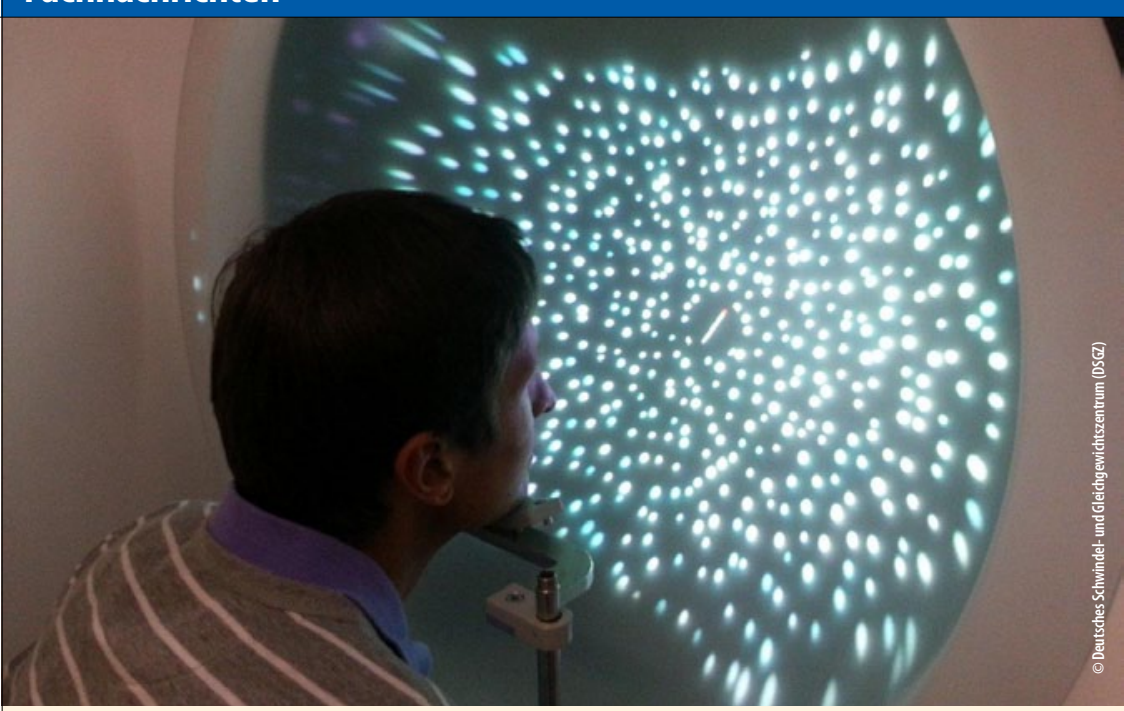

\section{VERTIGO XIX - Münchner Schwindel-Seminar 2015}

Periphere, zentrale und funktionelle Schwindelsyndrome

Am 10. und 11. Juli 2015 findet das inzwischen neunzehnte Münchner SchwindelSeminar unter der wissenschaftlichen Leitung von M. Strupp, M. Dieterich, R. Gürkov und T. Brandt statt. Es wird gemeinsam vom Deutschen Zentrum für Schwindel und Gleichgewichtsstörungen, der Neurologischen Klinik und der HNO-Klinik des Klinikums der Universität München veranstaltet.

Die Schwerpunkte dieser wie immer ganz klinisch orientierten Veranstaltung liegen am ersten Tag auf der Darstellung der häufigsten peripheren, zentralen und funktionellen Schwindelsyndrome. Die einzelnen Krankheitsbilder werden in jeweils 15 - bis 20-minütigen Kurzvorträgen mit den aktuellen diagnostischen Kriterien und Behandlungsmöglichkeiten beschrieben. Zusätzlich präsentieren wir Ihnen drei Fälle als Videoquiz. Am Ende des ersten Tages steht eine offene Diskussion mit allen Referenten.
Am zweiten Tag werden parallel Kurse mit praktischen Übungen stattfinden. Hier liegen die Schwerpunkte auf Okulomotorikstörungen, apparativen Untersuchungsverfahren und der Psychotherapie funktionellen Schwindels. Diese Kurse sind gleichermaßen geeignet für Neurologen, HNO- und Augenärzte, Orthoptistinnen und MTAs. Sie sollen in die körperlichen und apparativen Untersuchungstechniken sowie Therapieverfahren einführen und damit Kenntnisse und praktische Fertigkeiten vertiefen.

\section{Veranstalter:}

Deutsches Zentrum für Schwindel und Gleichgewichtsstörungen Neurologische Klinik und HNO-Klinik Klinikum der Universität München Campus Großhadern

Marchioninistraße 15, 81377 München

\section{Anmeldung:}

www.deutsches-schwindelzentrum.de Anmeldeschluss ist der 28. Juni 2015 